\title{
Retinoic acid-induced upregulation of miR-219 promotes the differentiation of embryonic stem cells into neural cells
}

\author{
Haibo $\mathrm{Wu}^{\star, 1,2}$, Jiamin Zhao ${ }^{1,2}$, Beibei Fu ${ }^{1,2}$, Songna Yin ${ }^{2}$, Chao Song ${ }^{1,2}$, Jingcheng Zhang ${ }^{1,2}$, Shanting Zhao ${ }^{1}$ and Yong Zhang ${ }^{\star, 1,2}$
}

MicroRNAs (miRNAs) regulate critical cell processes, such as apoptosis, proliferation, and development. However, the role of miRNAs in embryonic stem cell (ESC) neural differentiation induced by retinoic acid (RA) and factors that govern neural directional differentiation remain poorly understood. In this study, we demonstrated that miR-219 is sufficient in promoting mouse ESCs to undergo neural differentiation. We discovered that Foxj3 and Zbtb18, two target genes of miR-219, are not able to determine the process of RA-induced differentiation, however they prevent ESCs from differentiating into neural cells. We identified four downstream genes, namely, Olig1, Zic5, Erbb2, and Olig2, which are essential to the gene interaction networks for neural differentiation. These data explain the mechanism of RA-induced neural differentiation of mESCs on the basis of miRNAs and support the crucial role of miR-219 in neurodevelopment.

Cell Death and Disease (2017) 8, e2953; doi:10.1038/cddis.2017.336; published online 27 July 2017

MicroRNAs (miRNAs) are a class of non-coding, singlestranded, and 22-25-nucleotide RNA molecules that negatively regulate gene expression in plants and animals. ${ }^{1}$ MiRNAs mainly pair with the 3 '-untranslated regions (UTRs) of a target gene and consequently degrade the target mRNA or inhibit translation. MiRNAs are involved in the regulation of embryonic development, apoptosis, and proliferation. ${ }^{2-7}$ Moreover, some miRNAs, as oncogenes or tumor suppressor genes, are closely related to tumor formation. ${ }^{8-11}$

All-trans retinoic acid (RA), a metabolic compound derived from Vitamin $A$, is important in cellular differentiation and neurogenesis. ${ }^{12-17} \mathrm{RA}$ is the most used morphogen for producing neural progenitor cells and neurons from pluripotent stem cells in vitro. ${ }^{18-21}$ The action of RA on differentiation is probably mediated by RA receptors (RARs) in the nucleus. This action is done through the binding of the receptors to DNA sequences located in the promoter regions of RA-responsive genes. ${ }^{17,22,23}$ Furthermore, the molecular signaling pathways involved in neuronal differentiation are complex. The specific mechanism of RA-induced neural differentiation in pluripotent stem cells is not well characterized, and the role of miRNAs in this process remains unknown. ${ }^{24-26}$

Forkhead box J3 (Foxj3) belongs to a gene family of transcription factors that regulate skeletal muscle and peripheral artery developments. ${ }^{27,28}$ Meanwhile, zinc-finger and BTB domain containing 18 (Zbtb18), also known as Zfp238, is involved in skeletal muscle differentiation and myogenesis. ${ }^{29,30}$ When combined with PDGFRa and Sox6, Foxj3 and Zbtb18 can control oligodendrocyte differentiation and myelination. ${ }^{31}$ However, the roles of Foxj3 and Zbtb18 in stem cell differentiation are poorly understood. In this study, we demonstrated that miR-219 can promote embryonic stem cells (ESCs) to differentiate into neural cells, and Foxj3 and Zbtb18, as targets of miR-219, are dominant factors in neural differentiation. These results provide a theoretical basis for RA application in ESC research and make a contribution to the understanding of neural regulatory networks.

\section{Results}

RA-induced miRNA expression changes in ESCs. RA is widely used to induce neurogenesis. ${ }^{18-20}$ We determined whether or not miRNAs are involved in RA-induced neural directional differentiation by performing an array-based miRNA profiling on mouse J1 ESCs (gene expression omnibus (GEO) accession number: GSE54145; all tested miRNAs sorted by names are listed in Supplementary Data s03). The results showed that 43 miRNAs were upregulated and 281 miRNAs were downregulated after $48 \mathrm{~h}$ of RA treatment in ESCs. Of the 43 upregulated miRNAs (Supplementary Table S1), 18 exhibited more than a fivefold change, and five other miRNAs that are well known to be functionally related with development were then confirmed by qRT-PCR. ${ }^{32-34}$ Consistent with the microarray findings, the 18 changed miRNAs were upregulated by RA treatment at varying degrees(Figure 1a). Three of them, namely, miR-10a$5 p$, miR-219-5p, and miR-219-2-3p, showed highly significant fold changes. As previously reported, RA-induced differentiation of mESCs is powerful and irreversible, and the first $36-48 \mathrm{~h}$ of induction is critical. ${ }^{12}$ Consequently, the expression levels of these three significantly changed miRNAs were detected at different time points during RA induction

${ }^{1}$ College of Veterinary Medicine, Northwest A\&F University, 22 Xinong Road, Yangling 712100, China and ${ }^{2}$ Key Laboratory of Animal Biotechnology, Ministry of Agriculture, Northwest A\&F University, 3 Taicheng Road, Yangling 712100, China

${ }^{*}$ Corresponding author: H Wu or Y Zhang, College of Veterinary Medicine, Northwest A\&F University, Yangling 712100, China. Tel/Fax: +86 29 87080092; E-mail: hbwu029@nwsuaf.edu.cn or zhangy1956@sina.com

Received 22.3.17; revised 21.5.17; accepted 14.6.17; Edited by y Shi 


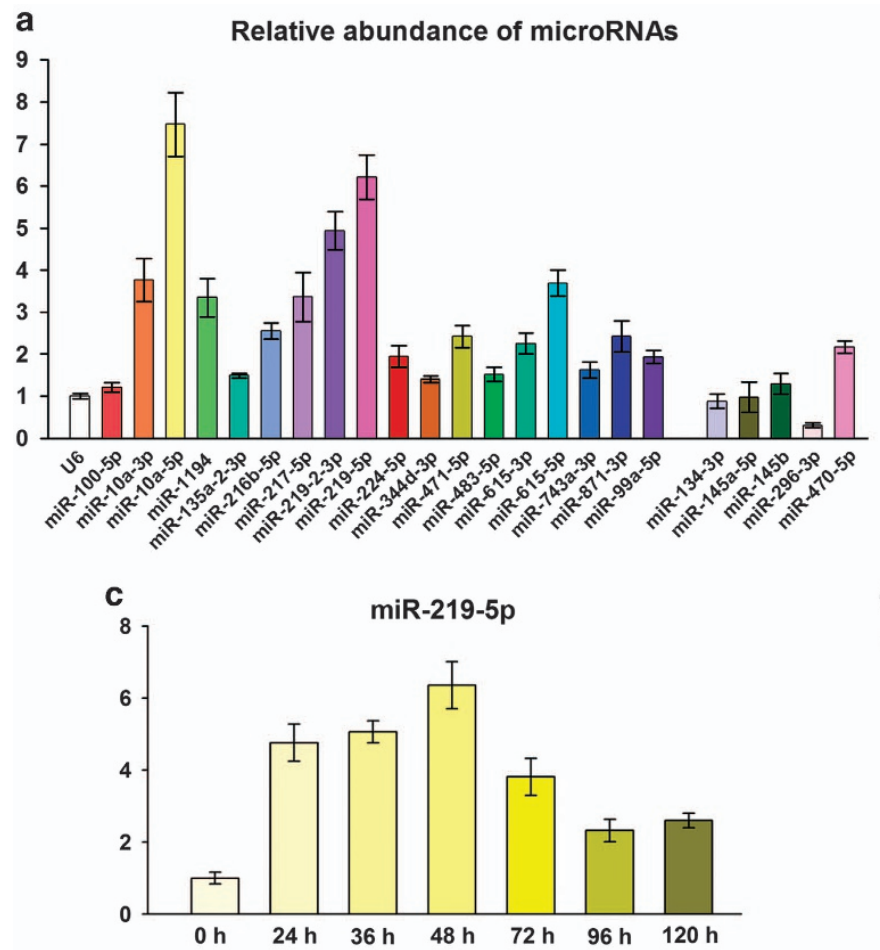

b
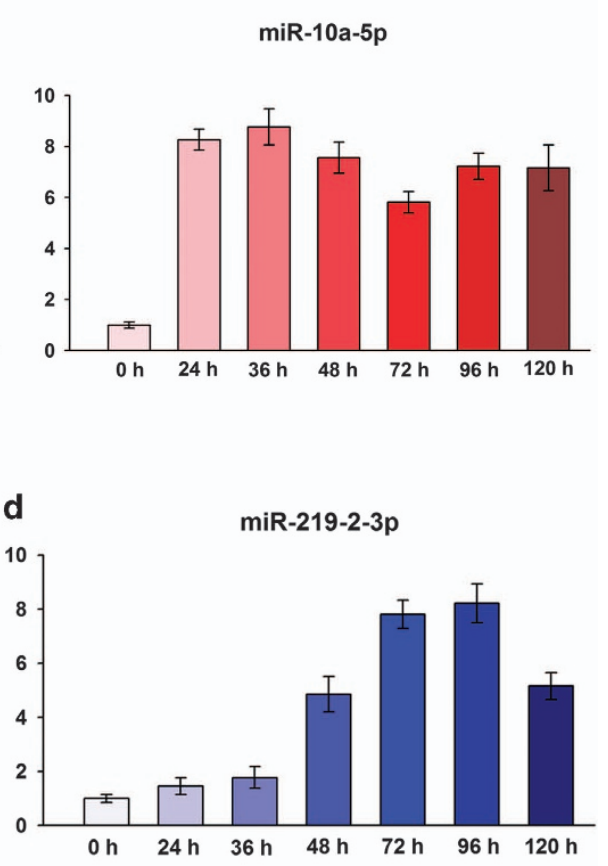

Figure 1 MiRNA expression changes in J1 ESCs induced by RA. (a) J1 ESCs were treated with RA for $48 \mathrm{~h}$, and qRT-PCR was performed to determine the relative expression levels of 23 miRNAs. U6 served as the internal control. (b-d) Relative expression levels of miR-10-5p (b), miR-219 (c), and miR-219-2-3p (d) at different time points after RA treatment on ESCs. All data are presented as mean \pm S.D. and derived from three independent experiments

(Figures 1b-d). The results showed that miR-219 was dramatically and highly expressed during the initial $48 \mathrm{~h}$, and its expression decreased gradually over time (Figure 1c). This result was verified in D3 and B6 ESCs (Supplementary Figure S1A), suggesting that miR-219 plays an important role in RA-induced ESC differentiation.

MiR-219 mediates ESCs to differentiate into neural cells. Next, miR-219 mimics and inhibitors were used to investigate whether or not miR-219 is involved in RA-induced differentiation. The validity of miR-219 mimics and inhibitors were verified in Figure $2 a$. As expected, the mESCs differentiated into neural-type cells $48 \mathrm{~h}$ after transfection of miR-219 mimics. The stem cell marker Oct4 was reduced considerably, whereas Nestin (a marker for neural stem cells), and Map2 and Tuj1 (markers for neural cells) were increased (Figures 2b-d). Furthermore, the ESCs transfected with miR-219 inhibitors resisted the RA-induced neural differentiation. As shown in Figures $2 \mathrm{e}-\mathrm{g}$, the miR-219 inhibitors blocked the RA-induced upregulation of Nestin, Map2, and Tuj1. The ESCs were differentiated after RA treatment regardless of the presence of miR-219 inhibitors, as characterized by the decreased Oct4 and loss of tight colony morphology (Figure $2 \mathrm{~g}$ ). Interestingly, the miR-219 inhibitors prevented ESCs from differentiating in a neural directional manner (Figures 2e-g; Supplementary Figures S1B-E). These findings suggested that miR-219 mediates ESCs to differentiate into neural cells.
Foxj3 and Zbtb18 are the targets of miR-219. To investigate the underlying mechanism, we examined the potential targets of miR-219 by searching the PicTar, miRanda, and targetScan databases. Among the candidate targets, the 3'-UTR of mouse Foxj3 and Zbtb18 contains putative regions that match the miR-219 seed sequence, which is conserved in humans and rats (Figure $3 a$ ). To confirm the predicted results, the $3^{\prime}$-UTRs of Foxj3 and Zbtb18 containing the putative regions were amplified and inserted into the psicheck-2 vector. They were then transfected to NIH/3T3 fibroblast cell line for dual luciferase reporter (DLR) assays. As shown in Figure $3 \mathrm{~b}$, the miR-219 mimics dramatically suppressed the activities of wild-type (WT) 3'-UTRs of Foxj3 and Zbtb18. By contrast, the double mutation type (MUT) group (site $1+2$ mut) with mutated seed sequences was unaffected. qRT-PCR and western blot were performed to examine the mRNA and protein levels of Foxj3 and Zbtb18 in ESCs transfected with miR-219 mimics or inhibitors. The results showed that the miR-219 mimics considerably decreased the protein levels of Foxj3 and Zbtb18 rather than the mRNA levels of these genes (Figures $3 c-e)$. Thus, miR-219 regulates the expression levels of Foxj3 and Zbtb18 at the post-transcriptional level. These results indicated that Foxj3 and Zbtb18 are the targets of miR-219.

Foxj3 and Zbtb18 prevent ESCs from differentiating into neural cells. MiR-219 promotes the neural differentiation ESCs (Figures 2e-g), targeting Foxj3 and Zbtb18 (Figures $3 b-d$ ). We then investigated whether or not Foxj3 

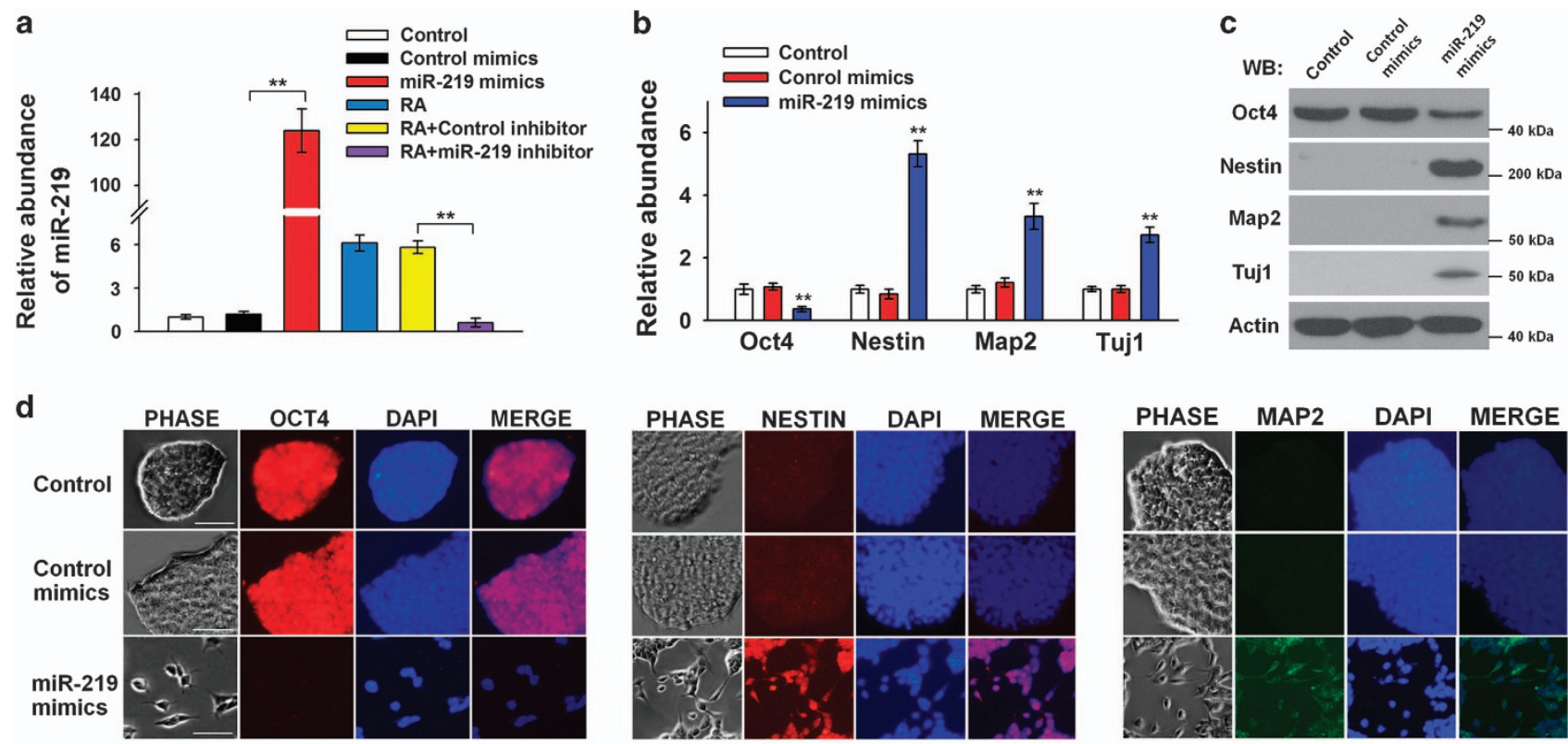

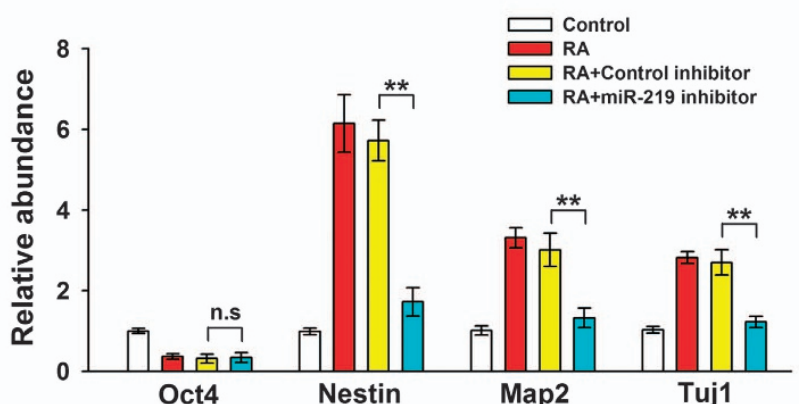

f

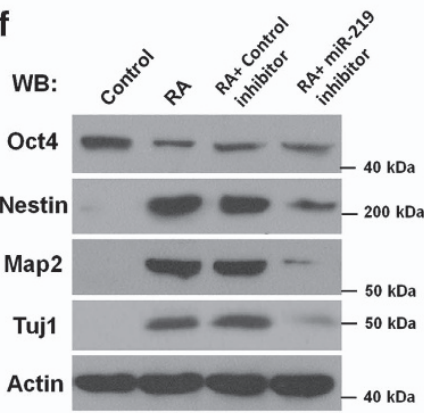

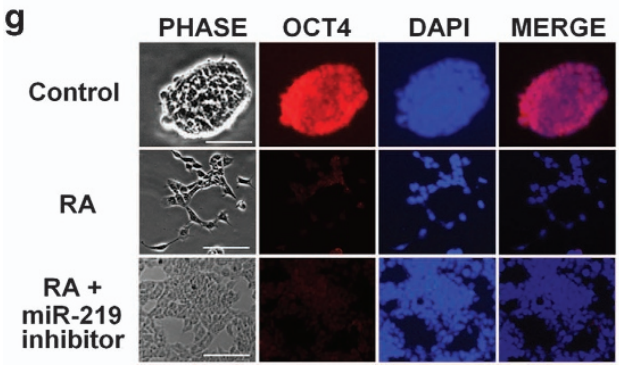
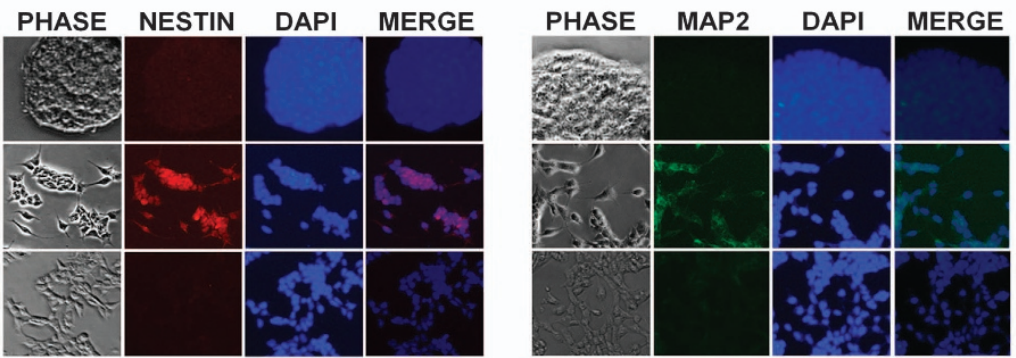

Figure 2 MiR-219 mediates ESCs to differentiate into neural cells. (a) Confirmation of the effects of miR-219 mimics and inhibitors. (b, c) ESCs were transfected with miR-219 mimics for $48 \mathrm{~h}$; the relative levels of Oct4, Nestin, and Map2 were detected through qRT-PCR (b) and western blot (c). (d) Immunofluorescence shows the abundance of Oct4, Nestin, and Map2, as well as the morphologies of the ESCs after transfection with miR-219 mimics for $48 \mathrm{~h}$. (e, f) ESCs were pretreated with RA for $48 \mathrm{~h}$ and transfected with miR-219 inhibitors. The relative levels of Oct4, Nestin, and Map2 were detected using qRT-PCR (e) and western blot (f). (g) Immunofluorescence shows the abundance of Oct4, Nestin, and Map2, as well as the morphologies of the ESCs pretreated with RA for $48 \mathrm{~h}$ and transfected with miR-219 inhibitors. ${ }^{\star \star} P<0.01$; NS, no significance

and Zbtb18 are involved in neural differentiation. Foxj3 and Zbtb18 were transiently transfected to ESCs, and the relative abundance of Nestin was detected. As expected, the Foxj3 or Zbtb18 disrupted the upregulation of Nestin after the miR-219 mimics treatment. Particularly, the synergistic effect of Foxj3 and Zbtb18 returned Nestin expression to basal levels (Figures 4a and b; Supplementary Figures S1F-K). Knockdown experiments were then conducted with small interfering RNA (siRNA) to verify the results. The results showed that Nestin expression was upregulated from 3.5 - to 4.5 -fold when
Foxj3 or Zbtb18 was knocked down, and knockdown of both Foxj3 and Zbtb18 at one time intensified neural differentiation (Supplementary Figures 4C, D). These results suggested that Foxj3 and Zbtb18 prevent the differentiation of ESCs into neural cells. The ES cell lines that stably expressed Foxj3 and Zbtb18 were produced by pCDH-Puro-Foxj3/Zbtb18 lentivirus to investigate the functional roles of Foxj3 and Zbtb18 in neural differentiation. The resulting cell lines were used for in vitro differentiation under RA treatment. Compared with normal ESCs, Foxj3/Zbtb18-overexpressing (OE) ESCs 
a

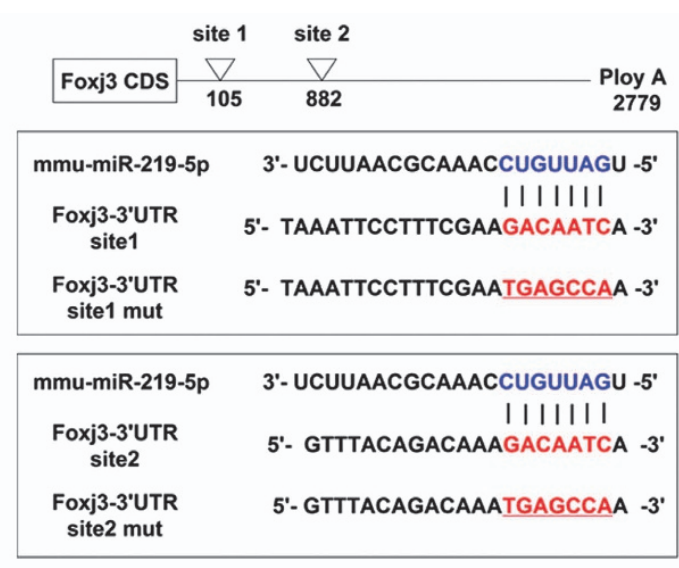

b

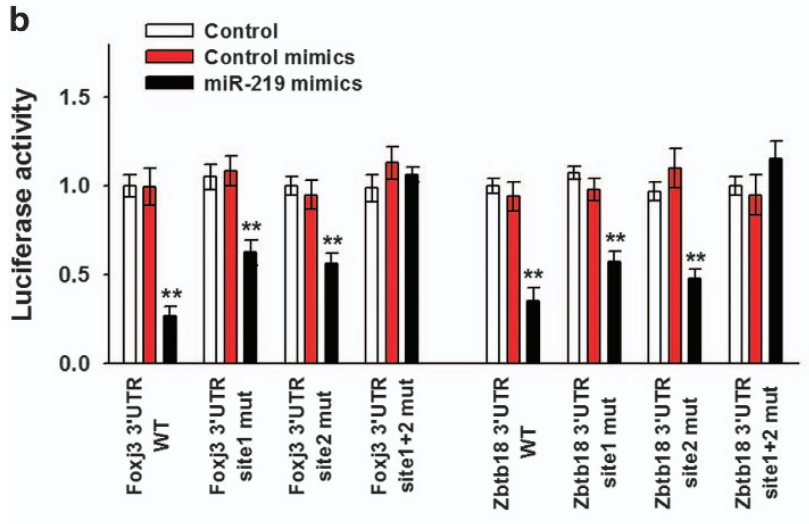

d

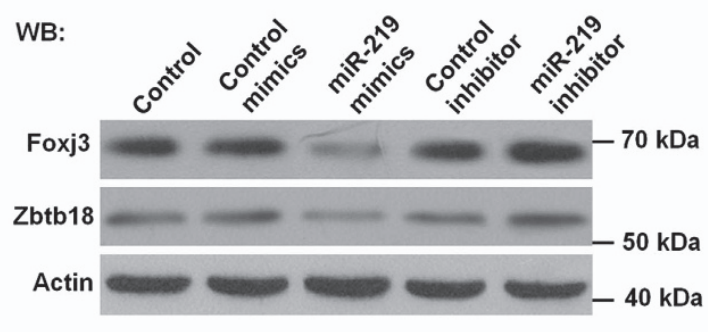

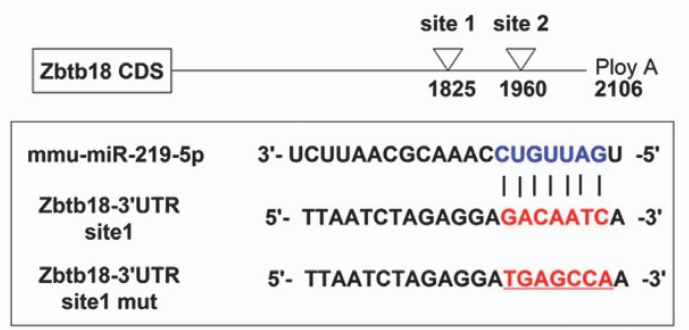

\begin{tabular}{|c|c|}
\hline mmu-miR-219-5p & $\begin{array}{l}\text { 3'- UCUUAACGCAAACCUGUUAGU -5' } \\
\qquad|||||| \mid\end{array}$ \\
\hline $\begin{array}{l}\text { Zbtb18-3'UTR } \\
\text { site2 }\end{array}$ & 5'- CATCTAGCTTTGACAATCA -3' \\
\hline $\begin{array}{l}\text { Zbtb18-3'UTR } \\
\text { site2 mut }\end{array}$ & 5'- CATCTAGCTTTTGAGCCAA -3' \\
\hline
\end{tabular}

c

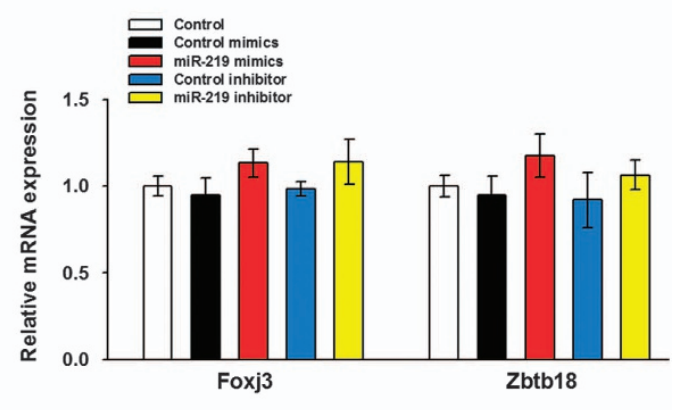

e

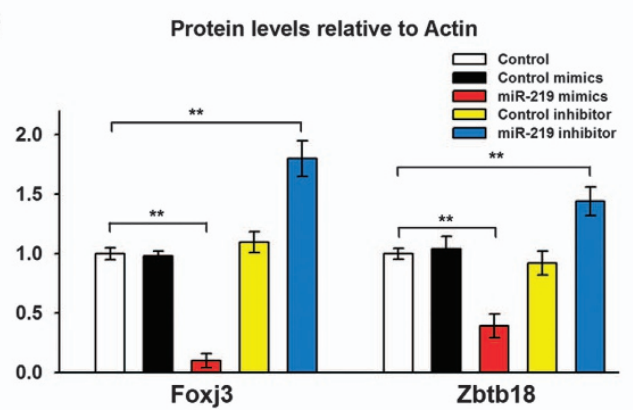

Figure 3 Foxj3 and Zbtb18 are the targets of miR-219. (a) 3'-UTR analysis of Foxj3 and Zbtb18 containing putative regions that match the seed sequence of miR-219. (b) At $24 \mathrm{~h}$ after NIH/3T3 fibroblast cells were transfected with miR-219 mimics, luciferase reporter constructs containing WT or MUT-type UTRs were transfected as indicated. Cell lysates were harvested for DLR assays. (c-e) MiR-219 mimics or inhibitors were transfected to ESCs. After $48 \mathrm{~h}$, cells were harvested for qRT-PCR (c) and western blot (d) to detect the relative levels of Foxj3 and Zbtb18. The relative intensities of the protein bands (e) were quantified with Image $\mathrm{J}$ software and calculated using the samples normalized to $\beta$-actin. All data are presented as mean \pm S.D. and derived from three independent experiments. Scale bars, $30 \mu \mathrm{m}$. ${ }^{\star \star} P<0.01$

differentiated but not in a neural directional manner, as characterized by morphology and the expression levels of neural markers Tuj1 and NeuN (Figure 4e).

Teratoma formation tests in nude mice were performed to confirm this finding. The Foxj3/Zbtb18-OE ESCs were pretreated with RA for $48 \mathrm{~h}$ and injected into nude mice to generate teratomas. The teratomas were harvested 14 days later for further examination. The hematoxylin/eosin- (H\&E) stained samples showed that the RA-pretreated ESCs tend to form neuronal-like tissues (Supplementary Table S2, pretreated $38.9 \%$ versus untreated $12.2 \%$ ), and the neuronal differentiation potential of ESCs was mostly suppressed by
Foxj3 and Zbtb18 (Supplementary Table S2, pretreated Foxj3/ Zbtb18-ESC 5.56\% versus pretreated ESC 38.9\%; representative neural-like tissues are shown in Supplementary Figure S2). Moreover, the Foxj3/Zbtb18-ESCs pretreated with RA retained the ability to form epidermis-like tissues (Figure 4f), suggesting that Foxj3 and Zbtb18 affected neuroectodermal development rather than the development of the entire ectoderm. In addition, the Foxj3/Zbtb18-OE ESCs generated less ectoderm-specific tissues $(21.1 \%$ versus $52.2 \%)$ and more mesoderm-specific (24.4\% versus $13.3 \%)$ and endoderm-specific $(28.9 \%$ versus $15.6 \%)$ tissues (Supplementary Table S2) compared with RA-pretreated 
a

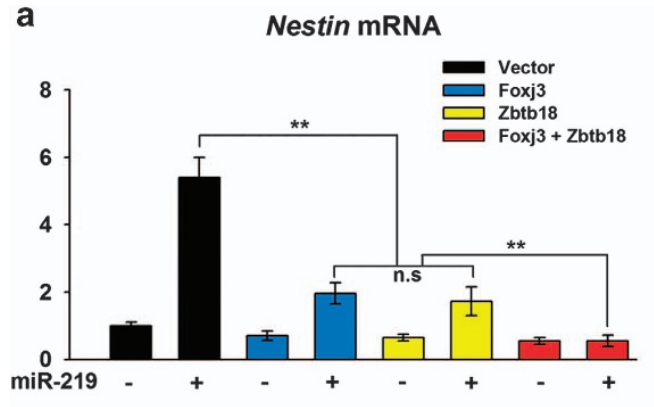

b

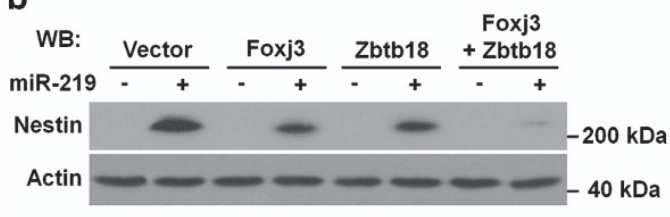

c
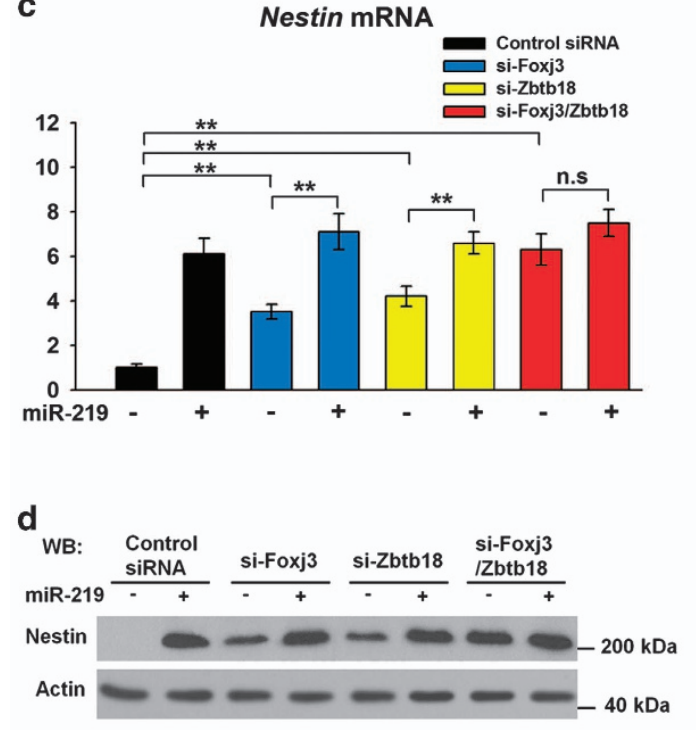

e

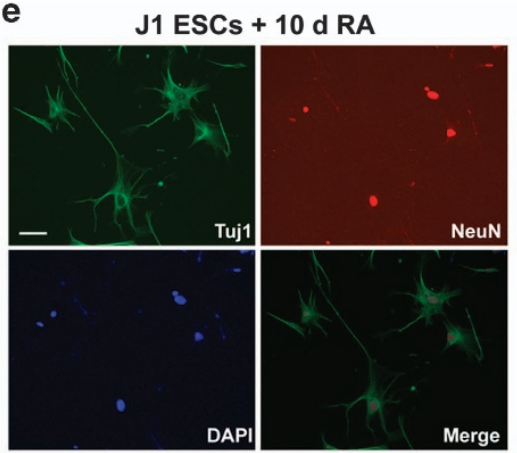

Foxj3/Zbtb18-ESCs + 10 d RA

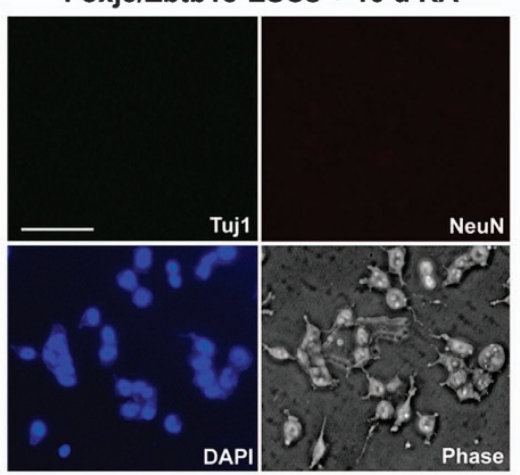

f

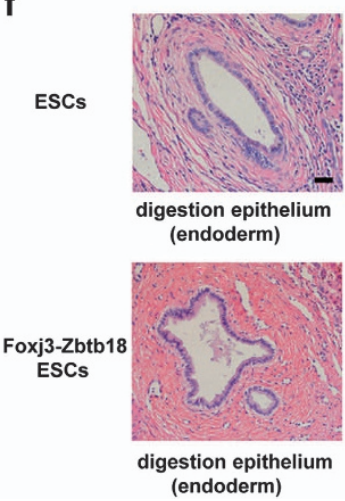

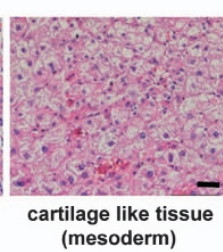
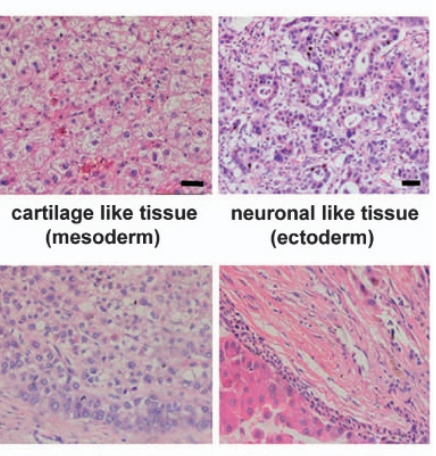

cartilage like tissue (mesoderm)

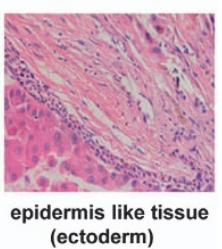

Figure 4 Foxj3 and Zbtb18 prevent ESCs from differentiating into neural cells. (a, b) ESCs were pretreated with miR-219 mimics for $24 \mathrm{~h}$ and followed by transfection of pCMV-Foxj3 or pCMV-Zbtb18 as indicated. After $48 \mathrm{~h}$, the relative level of Nestin was detected through qPCR (a) and western blot (b). Actin served as the loading control. (c, d) ESCs were pretreated with miR-219 mimics for $24 \mathrm{~h}$ and followed by transfection of Foxj3-siRNA or Zbtb18-siRNA. After $48 \mathrm{~h}$, the relative level of Nestin was detected through qPCR (c) and western blot (d). (e) Foxj3/Zbtb18-OE ESCs were induced by RA for 10 days. The morphology was observed and photographed. Scale bars: $100 \mu \mathrm{m}$. (f) Control ESCs or Foxj3/Zbtb18-OE ESCs were pretreated with RA for $48 \mathrm{~h}$ and injected into nude mice to generate teratomas. The differentiations of the three germ layers were analyzed through H\&E staining. Scale bars: $100 \mu \mathrm{m} .{ }^{* *} P<0.01$; NS, no significance

ESCs. We then tested the expression levels of mesodermspecific (Brachyury, Flk1) and endoderm-specific (Sox17, Foxa2) markers in ESCs treated with RA or miR-219 antagomirs. The results showed that the increase in the expression levels of the mesoderm- and endoderm-specific markers in ESCs transfected with miR-219 antagomirs were larger than that in the RA-treated cells (Supplementary Figure S3). These data indicated that RA-induced ESCs tend to differentiate into mesodermal and endodermal cells upon miR-219 inhibition.

To elucidate the role of miR-219-Foxj3/Zbtb18 in the regulation of neural induction in vivo, we examined the effects of miR-219 and Foxj3/Zbtb18 on developing embryos between egg-cylinder and primitive streak stages. MiR-219 agomirs or Foxj3/Zbtb18 mRNAs were injected into the zygote cytoplasm. Microinjected embryos that reached the blastocyst stage were transferred to pseudopregnant female recipients. The Oct4 and Nestin expression patterns in the serially sectioned E6.5E7.5 embryos were then examined by immunohistochemistry analysis (Figure 5). At E6.5, Nestin expression in the miR-219injected embryos occurred earlier than the control group (Figures 5A,b and d). At E7.5, the differences between the control and miR-219-injected embryos became more apparent. Approximately $27.8 \%(15 / 54)$ of the miR-219-injected 
A

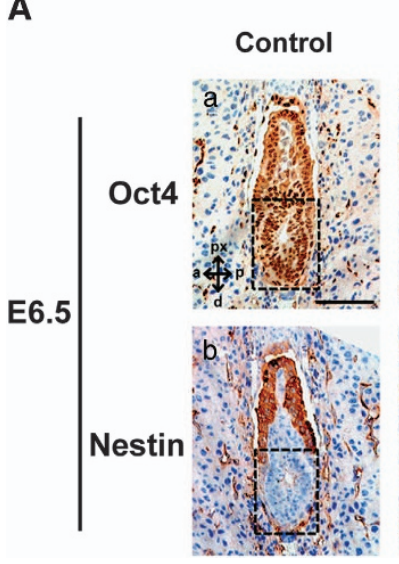

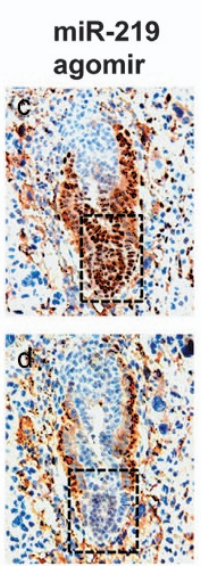

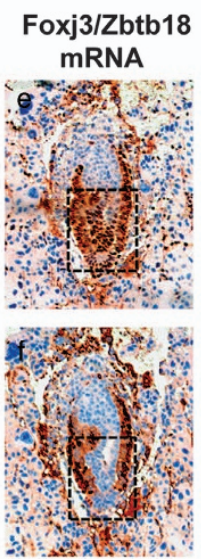

B

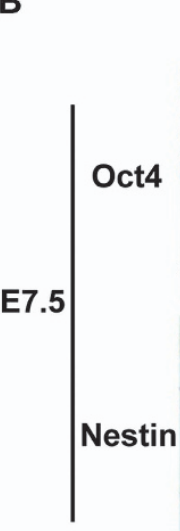

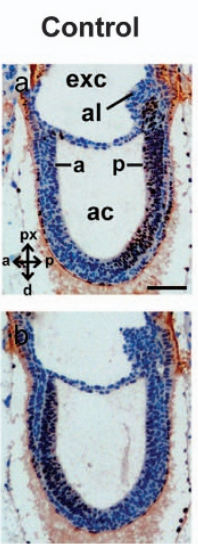

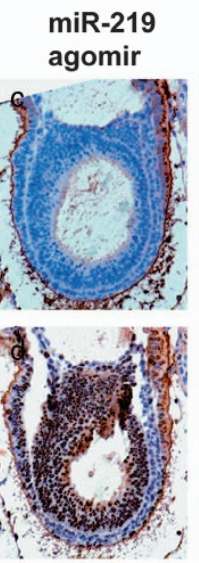

Foxj3/Zbtb18 mRNA

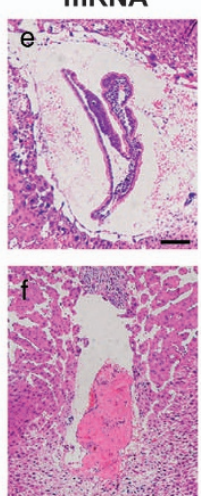

Figure 5 MiR-219 induces neural development in mouse embryos. MiR-219 agomirs, Foxj3/Zbtb18 mRNAs, and their corresponding negative controls were microinjected into the cytoplasm of zygotes as indicated. The expression patterns of Oct4 and Nestin in the serially sectioned E6.5 and E7.5 embryos were examined by immunohistochemistry analysis. (A) Immunohistochemistry analysis of the E6.5 embryos. Representative images are shown. Black dashed frames in E6.5 embryos indicate the embryonic region. (B) Immunohistochemistry $(a-d)$ and H\&E staining $(e, f)$ analysis of E7.5 embryos. a and b show the representative control embryo and indicate how the embryonic region is organized and how the ectodermal and endodermal tissues can be distinguished. $c$ and d show the representative abnormal miR-219-injected embryo, which exhibits a severe overdevelopment in its anterior region. (e, f) show representative abnormal Foxj3/Zbtb18 mRNA-injected embryo with disorganized embryonic region, apparent arrested development, or resorption. Full images of serially sectioned embryos are shown in Supplementary Figure S4. a, anterior; ac, amniotic cavity; al, allantois; d, distal; exc, exocoelomic cavity; p, posterior; px, proximal. Scale bars, $200 \mu \mathrm{m}$

embryos were resorbed or had no significant progress in their development, and $38.9 \%(21 / 54)$ of the embryos showed varying degrees of overdevelopment in the anterior region. This finding was supported by the immunohistochemistry analysis results. In the WTembryos, Oct4 was restricted in the posterior region, and Nestin was expressed in the anterior region because of forebrain formation (Figures 5B, $a$ and $b$ ). However, Oct4 disappeared and Nestin was detected in the entire ectoderm of the miR-219-injected embryos (Figures 5B, $c$ and d). These results indicated that miR-219 induces neural development in the mouse embryos. On the contrast, embryos injected with Foxj3/Zbtb18 mRNAs formed egg cylinders at E6.5 (Figures 5A, e and f), but 48.8\% (40/82) of the sectioned embryos showed quick degeneration and various abnormal phenotypes, $23.2 \%(19 / 82)$ of the embryos were resorbed completely or left a trace of residual pyknotic tissue by E7.5 (Figure 5B, e and f; Supplementary Figure S4). The extremely high rate of mutants in the miR-219- and Foxj3/Zbtb18injected embryos implied that the normal expression of Foxj3/ Zbtb18 is crucial in early embryonic development in mice.

Olig1 and Olig2 are important in miR-219-mediated neural differentiation. The total mRNAs of Foxj3-ES and Zbtb18-ES were extracted and used for RNA-seq and transcriptome analyses to understand the specific mechanism by which Foxj3 and Zbtb18 control neural differentiation of ESCs. Compared with control, 1331 genes were differentially expressed $(P<0.05)$ by two-fold in the Foxj3-ESCs $(557$ were underexpressed and 774 were overexpressed), and 1175 genes were differentially expressed $(P<0.05)$ by twofold in Zbtb18-ESCs (548 were underexpressed and 627 were overexpressed). Gene ontology (GO) analysis indicated that $53 \mathrm{GO}$ terms in Foxj3-ES and 54 GO terms in Zbtb18-ES were enriched $(P<0.01$; Supplementary Figures S5A, B). Foxj3 and Zbtb18 work synergistically to control neural differentiation, and thus the genes located in the four overlapping biological processes (i.e., nervous system development, central nervous system development, neuron fate commitment, and forebrain development) of the enriched GO terms were selected for analysis. A total of 37 candidate genes in the Foxj3-ES group and 39 candidate genes in the Zbtb18-ES group were present. Between these groups, 17 genes overlapped, namely Arid1a, Camk2b, Chd7, Erbb2, Glis2, Gnao1, Id2, Ina, Map1s, Ndn, Numbl, Olig1, Olig2, Shank1, Smarcd3, Zic2, and Zic5. We hypothesized that these genes contribute to RA-mediated neural differentiation. Their expression levels were verified through qRT-PCR (Supplementary Figure S5C; Supplementary Table S3).

To locate the core regulatory genes involved in neural regulatory networks, gene co-expression networks were constructed with the overlapping genes in the Foxj3-ES and Zbtb18-ES (Figure 6a). Core regulatory factors were then identified on the basis of the degree of differences between the control and Foxj3/Zbtb18-ES groups. The genes with a difference degree of more than eight were selected. Five genes, namely, Olig1, Zic5, Erbb2, Numbl, and Olig2, met the specification in the Zbtb18-ES group. Meanwhile, eight genes, namely, Olig1, Shank1, Erbb2, Zic5, Smarcd3, Ina, Chd7, and Olig2, met the specifications in the Foxj3-ES group. In addition, the following genes were present in both groups: Olig1, Zic5, Erbb2, and Olig2 (Figures 6b and c). We then performed qRT-PCR to validate the relationships of these genes with miR-219, Foxj3, and Zbtb18. As expected, Olig1, Zic5, Erbb2, and Olig2 were upregulated two-fold to five-fold when the ESCs were induced by RA treatment or miR-219 mimics, whereas they were dramatically inhibited when Foxj3 or Zbtb18 was overexpressed (Figure 6d). These findings were consistent with the RNA-seq results. To understand the roles of Foxj3, Zbtb18, and the four genes involved in transcription factor networks during RA-dependent neural 


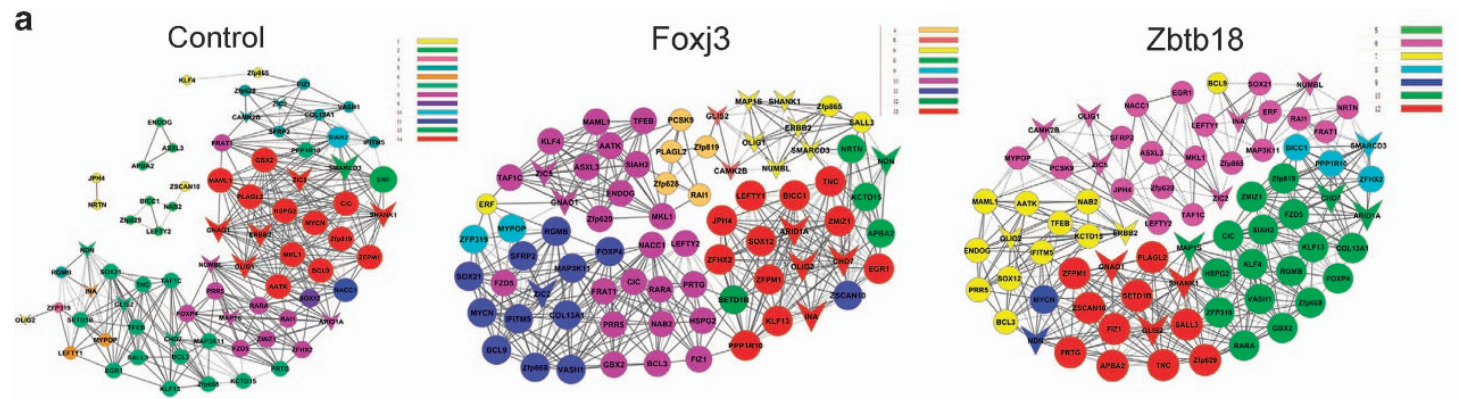

b

\begin{tabular}{cccccc}
\hline \multirow{2}{*}{ Gene } & \multicolumn{2}{c}{ Control } & \multicolumn{2}{c}{ Foxj3 } & \multirow{2}{*}{$\begin{array}{c}\text { Dif } \\
\text { degree }\end{array}$} \\
\cline { 2 - 5 } & Degree & K-core & Degree & K-core & -12 \\
\hline Olig1 & 20 & 14 & 8 & 6 & -12 \\
Shank1 & 19 & 14 & 7 & 6 & -11 \\
Erbb2 & 19 & 14 & 8 & 6 & -9 \\
Zic5 & 20 & 14 & 11 & 10 & -8 \\
Smarcd3 & 17 & 13 & 9 & 6 & 8 \\
Ina & 9 & 6 & 17 & 13 & 10 \\
Chd7 & 10 & 7 & 20 & 13 & 16 \\
Olig2 & 1 & 1 & 17 & 13 & \\
\hline
\end{tabular}

d

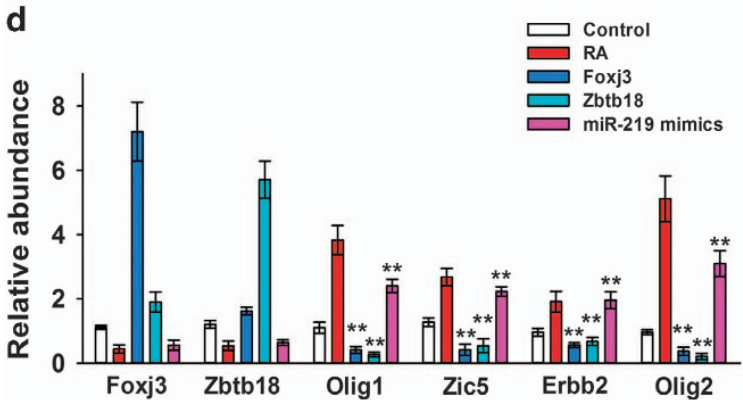

f

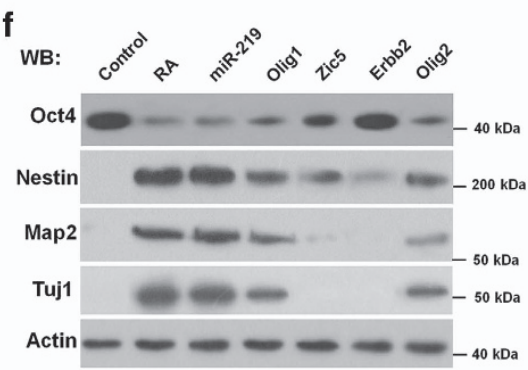

C

\begin{tabular}{cccccc}
\hline \multirow{2}{*}{ Gene } & \multicolumn{2}{c}{ Control } & \multicolumn{2}{c}{ Zbtb18 } & \multirow{2}{*}{$\begin{array}{c}\text { Dif } \\
\text { degree }\end{array}$} \\
\cline { 2 - 5 } & Degree & K-core & Degree & K-core & degrennnnnn \\
\hline Olig1 & 20 & 14 & 6 & 6 & -14 \\
Zic5 & 20 & 14 & 9 & 6 & -11 \\
Erbb2 & 19 & 14 & 11 & 7 & -8 \\
Numbl & 15 & 8 & 7 & 6 & -8 \\
Olig2 & 1 & 1 & 13 & 7 & 12 \\
\hline
\end{tabular}

e

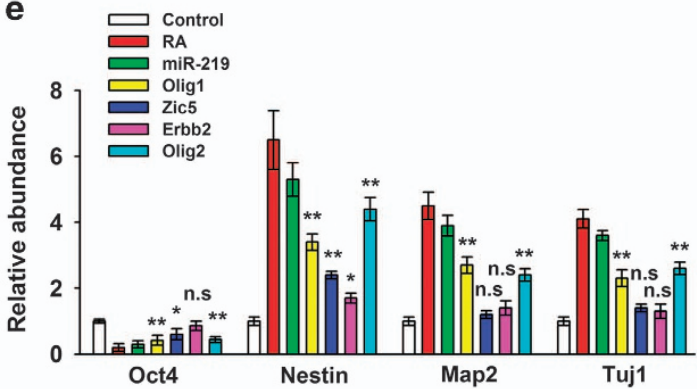

g

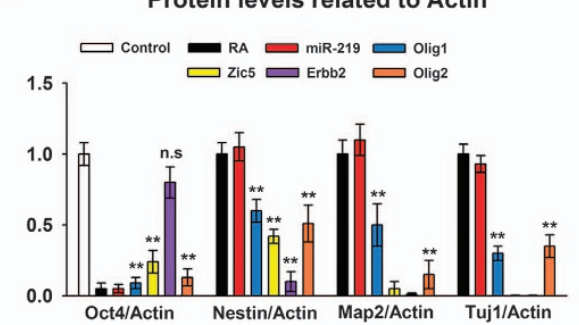

Figure 6 Olig1, Zic5, Erbb2, and Olig2 participate in neural regulatory networks. (a) Co-expression networks of differentially expressed genes in the control, Foxj3-ES, and Zbtb18-ES groups. The overlapping genes were selected to construct gene co-expression networks. Sixteen genes located in the four focused GO terms are arrow-shaped. Solid lines, positively corrected; dashed lines, negatively corrected. (b, c) The genes with difference degrees above eight between Foxj3-ES (b) or Zbtb18-ES (c) and control ESCs. (d) The relative levels of Olig1, Zic5, Erbb2, and Olig2 in the ESCs were detected through qRT-PCR. The ESCs were treated or transfected with RA, pCMV-Foxj3, pCMV-Zbtb18, or miR-219 for 48 h. (e-g) Relative levels of Nestin and Map2 were detected through qRT-PCR (e) and western blot (f) in ESCs transfected with Olig1-, Zic5-, Erbb2-, or Olig2encoding plasmids for $48 \mathrm{~h}$. Relative intensities of protein bands $(\mathbf{g})$ were quantified by using Image $\mathrm{J}$ software and calculated by using the samples normalized to $\beta$-actin. All data are presented as mean \pm S.D. and derived from three independent experiments. ${ }^{*} P<0.05$; ${ }^{*} P<0.01$; NS, no significance

differentiation, we examined the relative expression levels of these genes in the first 10 days of RA-induced differentiation (Supplementary Figure S6). The results showed that Foxj3 and Zbtb18 exhibited a similar expression pattern, that is, their expression levels rapidly declined in the first 2 days, and the temporary increased, and finally returned to a relatively low level during neural differentiation (Supplementary Figures S6A, B). However, the expression levels of Olig1 and Olig2 progressively increased and a sudden rise was observed around day 7 after RA-induced neural differentiation (Supplementary Figures S6C, F). This effect may be attributed to the presence of glial cells and neurons on that day.

Furthermore, we evaluated whether these four genes are involved in the neural differentiation. As shown in Figures $6 \mathrm{e}-$ $\mathrm{g}$, ESCs were differentiated into neural-type cells $48 \mathrm{~h}$ after expression of plasmids that encode Olig1, Zic5, Erbb2, or Olig2 were transfected, as characterized by the dramatic Oct4 reduction and Nestin increase. These results suggested that 


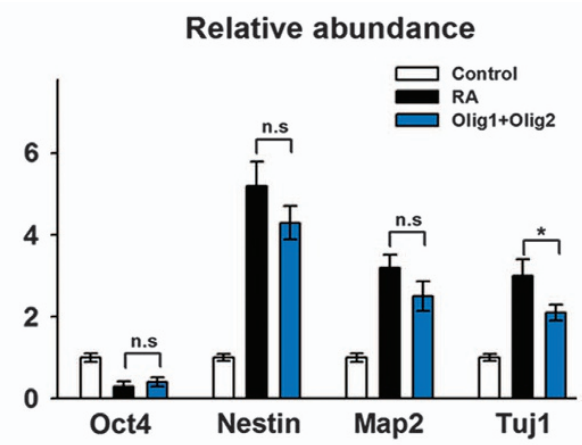

C

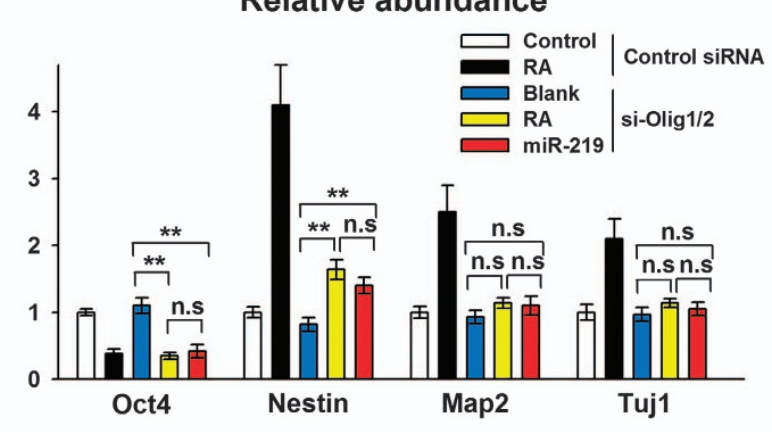

b

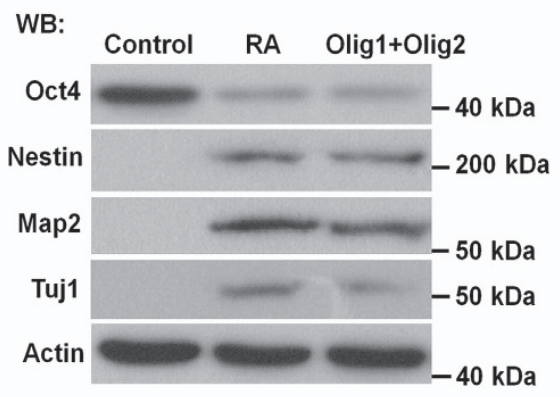

d

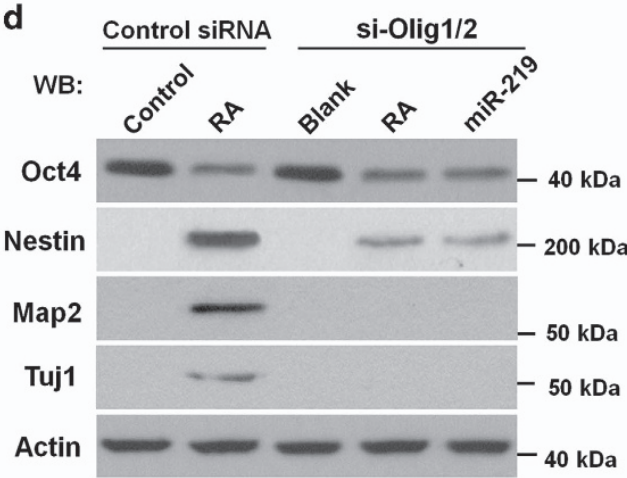

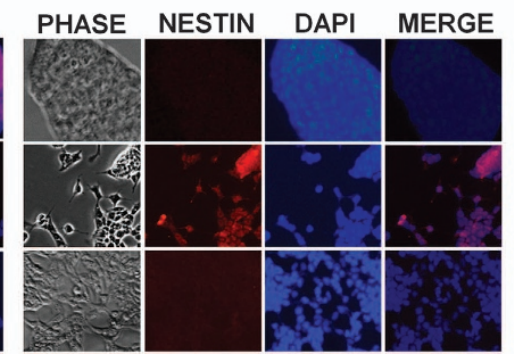

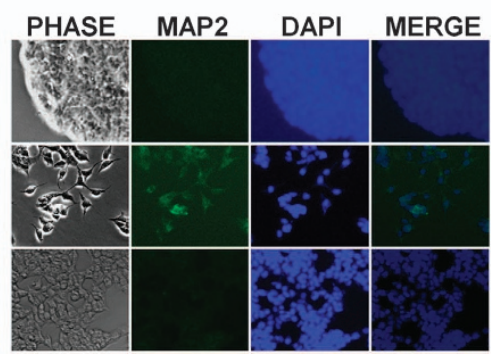

Figure 7 Olig1 and Olig2 are critical to miR-219-mediated neural differentiation. (a, b) Relative levels of Nestin, Map2, and Tuj1 were detected through qRT-PCR (a) and western blot (b) in ESCs transfected with Olig1/Olig2-encoding plasmids for $48 \mathrm{~h}$. (c, d) ESCs were transfected with siRNAs that were targeted to Olig1/Olig2 for $12 \mathrm{~h}$ and treated with RA or miR-219 mimics for another $36 \mathrm{~h}$. Relative levels of Nestin, Map2, and Tuj1 were detected through qRT-PCR (c) and western blot. (e) Immunofluorescence shows the abundance of Oct4, Nestin, and Map2, as well as the morphologies of ESCs transfected with Olig1/Olig2 or miR-291 mimics. ${ }^{* \star} P<0.01 ;$ NS, no significance

these four factors are involved in neural regulatory networks. Notably, only Olig1 and Olig2 facilitate the expression levels of Map2 and Tuj1 (neural cell markers) in the ESCs (Figures $6 \mathrm{e}-\mathrm{g})$. We hypothesized that Olig1 and Olig2 are the most dominant downstream factors of miR-219-mediated neural differentiation. Olig1/2 overexpression and knockdown were performed to demonstrate the involvement of Olig1 and Olig2 in the downstream of the RA-miR-219-Foxj3/Zbtb18 pathway. The results showed that Nestin, Map2, and Tuj1 dramatically increased in ESCs with overexpressed Olig1/Olig2, and the levels of neural differentiation were approximate to RA treatment (Figures 7a and b). Moreover, Olig1/2-knockdown ESCs cultured in the presence of RA or transfected with miR-219 mimics failed to differentiate into neural cells, as characterized by the reduction of Oct4 and absence of significant increase in neural cell markers (Map2 and Tuj1) (Figures 7c-e). These results indicated that Olig1 and Olig2 are the main factors regulated by miR-219 in RA-induced neural differentiation.

\section{Discussion}

RA can induce various types of cells in a concentrationdependent manner. For example, a high RA concentration increases the rate of neural differentiation, whereas a low RA concentration induces cardiomyocyte differentiation of mESCS. ${ }^{35}$ Okada et al. ${ }^{36}$ reported that RA concentration regulates dorsoventral identity, that is, high $\mathrm{RA}$ concentration induces a dorsal phenotype, and low RA concentrations induces a ventral phenotype during in vitro mESC differentiation. A similar phenomenon was observed in human pluripotent stem cells, in which low-dose RA increased hematopoietic progenitor cell generation, whereas low-dose RA abrogated blood cell generation in vitro. ${ }^{37}$ Here, we found that $1 \mu \mathrm{M}$ RA is sufficient to induce mESCs that cultured without any stromal cell line, neural culture medium, or neural growth factors undergo neural differentiation.

During early embryonic development, RA can facilitate embryonic positioning along the embryonic axis by acting as 
an intercellular signaling molecule that guides the development of the posterior portion of the embryo. ${ }^{18,38}$ Efforts have been undertaken to uncover the mechanism of the effects of RA in embryonic development. The most familiar mechanism is the binding of RA to RAR, which is bound to a DNA region called the RA response elements and affects the binding of other transcription factors. ${ }^{39-41}$ Moreover, RA acts through the Hox genes, which ultimately control the anterior and posterior patterns in early developmental stages. ${ }^{13} \mathrm{RA}$ also affects the changes in the epigenetic marks on histones and creates a heritable change in chromatin responsiveness. ${ }^{42-44}$ Kashyap et al. ${ }^{45}$ reported that coincident with the RA-induced transcriptional activation of the Hoxa and Hoxb cluster genes, the epigenomic configuration of these clusters is rapidly remodeled by $\mathrm{H} 3 \mathrm{~K} 4 \mathrm{me} 3$ and acH3. Notably, RA treatment stabilizes p53 in human ESCs, and p53 activates the expression levels of miR-34a and miR-145, which then repress the stem cell factors Oct4, KIf4, Lin28a, and Sox2 and accelerate ESC differentiation. ${ }^{46}$ These findings suggested that miRNAs are important in the regulation of RAassociated differentiation of ESCs.

To this end, we characterized the expression of miRNAs in mESCs with RA treatment for $48 \mathrm{~h}$. Our previous studies showed that ESCs start to differentiate irreversibly after RA pretreatment for $48 \mathrm{~h}$ and continued to do so even after RA withdrawal. ${ }^{12}$ Therefore, miRNAs with high expression levels during the early stage of RA induction may be critical in RAinduced neural directional differentiation of mESCs. We performed most of the experiments in J1 ESCs to investigate the miR-219-dependent neural regulatory networks, which was later confirmed in D3 and B6 ESCs and in C57BL/6 mice, to ensure that this finding is not a particular characteristic of a single cell line.

MiR-219 is necessary in promoting oligodendrocyte differentiation, and partially rescues oligodendrocyte differentiation defects caused by total miRNA loss. ${ }^{31}$ Kocerha et al. ${ }^{47}$ reported that miR-219 is important in the expression of behavioral aberrations associated with NMDA receptor hypofunction. Santa-Maria et al. ${ }^{48}$ found that miR-219 downregulation promotes neurodegeneration by targeting Tau.
Recent studies have suggested that miR-219 regulates NPC proliferation and differentiation. ${ }^{49,50}$ However, the specific role of miR-219 in RA-associated ESC differentiation is not elucidated. In this study, we found that miR-219 is sufficient in promoting neural differentiation by targeting Foxj3 and Zbtb18. On the basis of the results of the RNA-seq performed on Foxj3-ESCs and Zbtb18-ESCs, we constructed three gene co-expression networks and identified 17 core genes that are functionally related to neurodevelopment. Interestingly, 16 of these genes were upregulated during neural differentiation, showing that most factors that dominate neural differentiation can be induced. We then demonstrated that Foxj3 and Zbtb18 are the molecular switches in the neurodevelopment system. Furthermore, we identified four critical genes by constructing gene co-expression networks. Two of them, namely, Olig1 and Olig2 might be the most essential elements in neural differentiation of ESCs. Olig1/2-knockdown ESCs cultured in the presence of RA or transfected with miR-219 mimics failed to differentiate into neural cells, as characterized by the absence of increase of neural cell markers (Map2 and Tuj1). Notably, Nestin (neural stem cell marker) appeared in the Olig1/2-knockdown ESCs treated with RA or miR-219 mimics. This finding may be attributed to other factors or mechanisms involved in the miR-219-mediated neural differentiation apart from Olig1/2. However, this hypothesis requires validation through further experiments. Moreover, the mechanism of how RA upregulates miR-219 is not well understood. We speculate that histone demethylases accelerate DNA demethylation at the promoter region of miR-219, thereby increasing the expression level of miR-219 and facilitating neural differentiation. In addition to epigenetic modifications, other transcription factors can possibly mediate the effects of RA on miR-219 upregulation. ${ }^{51}$ Thus the involvement of histone demethylases or other transcription factors with the RA and miR-219 pathway is interesting for further investigation.

Overall, we discovered that functionally important gene expression changes mediated by miRNA contribute to RA-induced differentiation. We demonstrated that Foxj3 and Zbtb18, two target genes of miR-219, are the main controls in neural differentiation of ESCs (Figure 8). Our findings illustrate

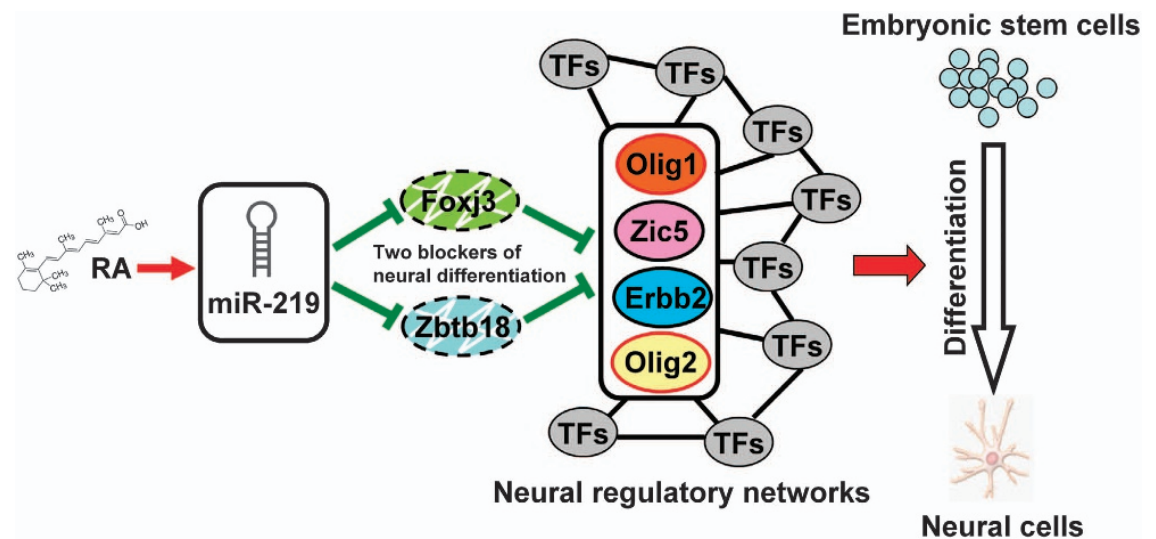

Figure 8 Schematic representation of RA-induced neural differentiation of ESCs. RA treatment led to upregulation of miR-219, which targeted Foxj3 and Zbtb18. Downregulation of Foxj3 and Zbtb18 resulted in the activation of Olig1, Zic5, Erbb2, and Olig2. The neural regulatory networks were triggered, and the neural directional differentiation of ESCs was determined. TF, transcription factor 
the mechanism of miRNA-mediated neural differentiation of ESCs, as well as the plasticity and dynamic nature of the gene regulatory networks during neural differentiation. However, the Foxj3 and Zbtb18 regulatory activities dependent on other transcription factors remain unclear. The specific mechanism of Foxj3 and Zbtb18 for determining the fate of neural differentiation and the functional roles of Foxj3 and Zbtb18 in multiple biological processes requires further investigation.

\begin{abstract}
Materials and Methods
Ethic statement. This study was carried out in strict accordance with the Guidelines for the Care and Use of Animals of Northwest A\&F University. All animal experimental procedures were approved by the Animal Care Commission of the College of Veterinary Medicine, Northwest A\&F University. C57BL/6 mice were purchased from Xi'an Jiao-tong University, China. Five-week-old female athymic nude mice were purchased from The Fourth Military Medical University. Every effort was made to minimize animal pain, suffering, and distress, and reduce the number of animals used.
\end{abstract}

Cell culture, transient transfection, and treatment. J1, R1, and D3 mESCs were purchased from American Type Culture Collection (ATCC, Manassas, VA, USA), and grown adherent to plastic plates (Corning Costar, Cambridge, MA, USA) coated with matrigel (Geltrex, Thermo Fisher Scientific, San Jose, CA, USA) and maintained under a feeder-free and serum-free system. The ESC culture medium was composed of Knockout Dulbecco's modified Eagle's medium (Knockout DMEM, Thermo Fisher Scientific), 15\% (v/v) Knockout Serum Replacement (Thermo Fisher Scientific), $1 \times$ non-essential amino acids (Thermo Fisher Scientific), $100 \mu \mathrm{M} \beta$-mercaptoethanol (Millipore, Bedford, MA, USA), $2 \mathrm{mM}$ glutamine (Thermo Fisher Scientific), 50 units $/ \mathrm{ml}$ penicillin, $50 \mu \mathrm{g} / \mathrm{ml}$ streptomycin, and 1000 units/ml LIF (ESGRO, Millipore). The growth condition of mESCs was consistent throughout this study.

Plasmids were transfected with Xfect Transfection Reagent (Clontech, Palo Alto, CA, USA) following the manufacturer's instructions. The transfection efficiency was monitored by a backbone vector with an extra eGFP element and calculated by flow cytometry. The average transfection efficiency was $\sim 75 \%-85 \%$, and the representative result is shown in Supplementary Figure S7. Double-stranded mmumiR-219-5p (miRBase accession number MIMAT0000664, 5'-UGAUUGUCCA AACGCAAUUCU-3') mimics, single-stranded mmu-miR-219-5p inhibitors and their corresponding negative controls were purchased from GenePharma (GenePharma, Shanghai, China). Negative control siRNA and siRNAs that target Foxj3, Zbtb18, Olig1, and Olig2 were chemically synthesized by Ribobio (Guangzhou, Guangdong, China). The knockdown efficiencies of siRNAs were verified by qRT-PCR and western blot (Supplementary Figure S8). The miRNAs and siRNAs were transfected with Lipofectamine RNAiMAX reagent (Invitrogen, Life Technologies, Carlsbad, CA, USA) following the manufacturer's instructions. RA (all-trans-Retinoic acid, product number: R2625) was purchased from Sigma-Aldrich (St. Louis, MO, USA). A stock solution of $1 \mathrm{mM}$ RA was prepared in absolute ethanol, stored at $-20^{\circ} \mathrm{C}$, and protected from light. The working concentration of RA was $1 \mu \mathrm{M}$. Culture medium and RA were changed daily and ESCs were passaged every $2-3$ days at a $1: 3-1: 5$ ratio according to cell density. A direct induction system without any stromal cell line, neural culture medium, or neural growth factors as neural inducer was performed to render the normal cultured ESCs a perfect control.

Construction of plasmids. Full-length coding sequences of Foxj3 (NCBI accession number NM_172699) and Zbtb18 (NCBI accession number NM_001012330) were amplified from J1 cDNA. These fragments were inserted into pCMV-HA (Clontech; verified in Supplementary Figure S8), pCDH-MCS-T2APuro-MSCV (System Biosciences, Palo Alto, CA, USA), and pCAG-EGFP (kindly provided by $\mathrm{Dr}$ Wei Zhang) vectors. The $3^{\prime}$-UTR of Foxj3 (1084 bp in length. Located at bases 2041-3124 refer to NM_172699.3; -34 to +1050 if consider base 2075 as the start site of UTR) and Zbtb18 (936 bp in length. Located at bases $2886-3821$ refer to NM_001012330.1; +1141 to + 2076 if consider base 1746 as the start site of UTR) were amplified from J1 cDNA and inserted into the psicheck-2 vector (Promega, Madison, WI, USA) through standard molecular cloning methods and confirmed by sequencing. All the primers used for plasmid construction are listed in Supplementary Tables S4 and S5.
Luciferase assays. Luciferase assays were performed using the DLR Assay System (Promega) as previously described. ${ }^{52}$ In brief, ESCs were cotransfected with a luciferase reporter construct and internal control plasmid pRL-SV40 (Promega). Cells were lysed $24 \mathrm{~h}$ after transfection or treatment, and relative luciferase activities were measured by firefly luciferase luminescence divided by Renilla luciferase luminescence.

Real-time qRT-PCR. We used qRT-PCR to analyze miRNA expression. Total RNA samples (including small RNA molecules) were isolated using mirVana miRNA Isolation Kit (Ambion, Austin, TX, USA). Purified RNA was reverse-transcribed using a miScript II RT Kit (Qiagen, Hilden, Germany). The expression of mature miRNAs was quantified using a miScript SYBR Green PCR Kit, which contained 10x miScript Universal Primer (Qiagen), and was performed according to the manufacturer's instructions. Quantization of $\mathrm{U} 6$ was performed to normalize miRNA expression levels.

We also used qRT-PCR to analyze gene expression. Total RNA samples were isolated using Trizol reagent (Invitrogen). Purified RNA was reverse-transcribed using a SYBR PrimeScript RT-PCR Kit (Takara, Otsu, Shiga, Japan). The expression of mRNAs was quantified using a SYBR Premix ExTaq II Kit (Takara). Real-time PCR was performed on an ABI StepOnePlus PCR system (Applied Biosystems, Foster City, CA, USA), and results were normalized to $\beta$-actin mRNA levels. Data were analyzed using the $2^{-\Delta \Delta C t}$ method. Primer sequences used for $\mathrm{qPCR}$ are listed in Supplementary Tables S6 and S7.

Western blot analysis. Western blot analysis was performed as previously described. ${ }^{52}$ Blots were probed with 1/1000 rabbit anti-Oct4 (\#2788), 1/1000 rabbit anti-MAP2 (\#4542) (Cell Signaling Technology, Beverly, MA, USA), 1/500 rabbit antiFoxj3 (\#SAB2100844), 1/500 rabbit anti-Zbtb18 (\#SAB2103436), 1/1000 mouse anti-Nestin (\#MAB5326), 1/1000 rabbit anti-Brachyury (\#B8436), 1/2000 mouse anti- $\beta$-actin (\#A5441) (Sigma-Aldrich), 1/1000 goat anti-Flk1 (VEGF receptor 2, \#AF644; R\&D Systems, Minneapolis, MN, USA), 1/1000 mouse anti-Sox17 (\#ab192453), 1/1000 rabbit anti-Foxa2 (\#ab108422) (Abcam, Cambridge, MA, USA), and 1/1000 mouse anti-Tuj1 (neuronal class III $\beta$-tubulin, \#MMS-435P; Covance, Princeton, NJ, USA). Immunoblots were revealed by autograph using SuperSignal west pico substrate (Thermo Fisher Scientific). The relative intensity of the protein bands was quantified using Image $\mathrm{J}$ software (NIH, Bethesda, MD, USA) and calculated by samples normalized to the controls. All data were presented as mean \pm S.D. and derived from three independent experiments.

RNA-seq and data analysis. Total RNA was extracted from Foxj3-ESCs or Zbtb18-ESCs by Trizol reagent (Invitrogen) separately. Then, the RNA samples were sent to Novel Bioinformatics company (Shanghai, China) for RNA-seq. The RNA quality was checked by Bioanalyzer 2200 (Agilent Technologies, Santa Clara, CA, USA) and kept at $-80^{\circ} \mathrm{C}$. The RNA with RIN $>8.0$ is right for CDNA library construction. RNA libraries were prepared for sequencing using lonProton. The CDNA libraries were processed for the proton sequencing process according to the commercially available protocols. Data were submitted to the GEO archive. The accession number is GSE61748. All tested genes sorted by fold changes are listed in Supplementary Data S04-S06. Pathway analysis was used to find out the significant pathway of the differential genes according to KEGG database. Fisher's exact test was calculated to select the significant pathway, and the threshold of significance was defined by $P$-value and false discovery rate $(\mathrm{FDR})^{53}$

GO analysis was performed to facilitate elucidating the biological implications of unique genes in the significant or representative profiles of the differentially expressed gene in the experiment. GO annotations were downloaded from NCBI (http://www. ncbi.nlm.nih.gov/), UniProt (http://www.uniprot.org/), and the GO (http://www. geneontology.org/). Fisher's exact test was applied to identify the significant GO categories and FDR was used to correct the $P$-values.

Gene co-expression networks were presented to find the relations among genes. Gene co-expression networks were built according to the normalized expression values of genes selected from genes in significant $\mathrm{GO}$ terms. For each pair of genes, we calculate the Pearson correlation and choose the significant correlation pairs $(F D R<0.05)$ to constructed the network. ${ }^{54}$ Degree centrality is defined as the link numbers one node has to the other. ${ }^{55}$ While considering genes in different networks, core regulatory factors were determined by the degree differences (Dif degree) between control ESCs and Foxj3/Zbtb18-ES. K-cores were also introduced to simplify the graph topology analysis. K-core means that at least $\mathrm{K}$ other genes are connected to a node. ${ }^{56,57}$ 
Teratoma formation, H\&E staining, and scoring method. The ESCs with stable expressions of Foxj3/Zbtb18 were pretreated with RA for $48 \mathrm{~h}$, and injected to nude mice to generate teratomas. The differentiations of the three germ layers were analyzed by H\&E staining. In brief, teratomas were fixed with $10 \%$ buffered formaldehyde for more than $24 \mathrm{~h}$, embedded in paraffin, sectioned, and stained with $H \& E$ according to the standard procedure. To evaluate the potential of three germ layer differentiation, we generated nine teratomas from the control ESCs, RA-pretreated ESCs, and RA-pretreated Foxj3/Zbtb18-OE ESCs, respectively. We obtained 10 slices from each teratoma, and each slice was observed by three operators. Individual germ layer-specific tissue scores were added to calculate the germ layer score. The scores of the nine teratomas were added to determine the percentages of three germ layer differentiations of the control ESCs, RA-pretreated ESCs, or RA-pretreated Foxj3/Zbtb18-OE ESCs. The scoring of all the slices was performed by the same operators for consistency.

Embryo preparation, microinjection, and immunohistochemistry analysis. For embryo preparation, C57BL/6 mice were maintained under controlled lighting conditions ( $12 \mathrm{~h}$ light : $12 \mathrm{~h}$ dark). After mating, the morning when the vaginal plug appeared was designated as embryonic day 0.5 (E0.5). Mouse zygotes were collected and denuded of cumulus cells. MiR-219 agomir, antagomir, and their corresponding negative controls were chemically synthesized (Ribobio) and diluted with a dilution buffer (Ribobio) to a final concentration of $5 \mathrm{nM}$. The Foxj3/Zbtb18 mRNAs were synthesized in vitro using the T7 Quick Coupled Transcription/Translation System (Promega) and adjusted to a final concentration of $50 \mathrm{ng} / \mu \mathrm{l}$ according to the manufacturer's instructions. Approximately $10 \mathrm{pl}$ of miR-219 agomirs or Foxj3/Zbtb18 mRNAs were injected into the cytoplasm of the zygotes under a Zeiss Axio Observer Z1 fluorescence microscope (Carl Zeiss, Jena, Germany) equipped with an Eppendorf Transfer-Man NK2 micromanipulator (Eppendorf, Hamburg, Germany). The microinjected embryos were cultured in M16 (Sigma-Aldrich) medium at $37^{\circ} \mathrm{C}$ at a humidified atmosphere containing $5 \% \mathrm{CO}_{2}$ to enable their development to the blastocyst stage.

Pseudopregnant female mice (recipients) were mated with vasectomized males 3.5 days prior to embryo transfer. Well-developed blastocysts with similar morphologies were then selected for embryo transfer in each group. Twelve blastocysts were transferred to each recipient, with six embryos in each uterine horn.

Recipients were killed 3 or 4 days after embryo transplantation to recover E6.5 or E7.5 embryos. The embryos were dissected in DMEM with $10 \%$ FBS. The conceptuses covered with decidual mass were extracted from the uterus and fixed in $4 \%$ paraformaldehyde. The embryos were then processed for paraffin wax embedding. Then, sections with thickness of $5 \mu \mathrm{m}$ were obtained, dewaxed in xylene, and rehydrated through an ethanol series into PBS. The sections were incubated with mouse anti-Nestin (1:100, \#MAB5326; Sigma-Aldrich) or rabbit anti-Oct4 antibody (1 : 100, \#SAB2701972; Sigma-Aldrich) at $4{ }^{\circ} \mathrm{C}$ overnight, and then incubated with horseradish peroxidase-conjugated secondary antibody at room temperature for $2 \mathrm{~h}$. Photographs were obtained with fluorescent microscope (Carl Zeiss).

Statistical analysis. All data are presented as mean \pm S.D. and derived from at least three independent experiments. Statistical significances were analyzed using the Student's $t$-test. A value of $P<0.05$ was considered significant.

Data availability. All data in this paper have been deposited to the GEO of NCBI. The accession numbers are GSE54145 and GSE61748.

\section{Conflict of Interest}

The authors declare no conflict of interest.

Acknowledgements. We would like to thank Dai Chen, Mingfei Feng, and Lianle Bian of Novel Bioinformatics company for their technical assistance during the conduct of bioinformatics analysis; Jiaxing Lv and Jiong Chen for their contribution in plasmid construction, and Prof. Zhang of the Fourth Military Medical University for his assistance in nude mice feeding and H\&E analysis. This work was funded by State Key Program of National Natural Science Foundation of China (No. 31530075) and China Postdoctoral Science Foundation Grant (No. 2016M590978).

\section{PUBLISHER'S NOTE}

Springer Nature remains neutral with regard to jurisdictional claims in published maps and institutional affiliations.

1. Bartel DP. MicroRNAs: genomics, biogenesis, mechanism, and function. Cell 2004; 116 281-297.

2. Ambros V. The functions of animal microRNAs. Nature 2004; 431: 350-355.

3. Kloosterman WP, Plasterk RH. The diverse functions of microRNAs in animal development and disease. Dev Cell 2006; 11: 441-450.

4. Sun K, Lai EC. Adult-specific functions of animal microRNAs. Nat Rev Genet 2013; 14 535-548.

5. Miska EA. How microRNAs control cell division, differentiation and death. Curr Opin Genet Dev 2005; 15: 563-568.

6. He L, Hannon GJ. MicroRNAs: small RNAs with a big role in gene regulation. Nat Rev Genet 2004; 5: 522-531.

7. Le MT, Xie H, Zhou B, Chia PH, Rizk P, Um M et al. MicroRNA-125b promotes neuronal differentiation in human cells by repressing multiple targets. Mol Cell Biol 2009; 29 : 5290-5305.

8. Esquela-Kerscher A, Slack FJ. Oncomirs - microRNAs with a role in cancer. Nat Rev Cancer 2006; 6: 259-269.

9. Hao J, Zhao S, Zhang Y, Zhao Z, Ye R, Wen J et al. Emerging role of microRNAs in cancer and cancer stem cells. J Cell Biochem 2014; 115: 605-610.

10. Volinia S, Calin GA, Liu CG, Ambs S, Cimmino A, Petrocca F et al. A microRNA expression signature of human solid tumors defines cancer gene targets. Proc Natl Acad Sci USA 2006; 103: 2257-2261

11. Jiang $Y$, Yin $L$, Jing $H$, Zhang $H$. MicroRNA-219-5p exerts tumor suppressor function by targeting ROBO1 in glioblastoma. Tumour Biol 2015; 36: 8943-8951.

12. Wu H, Wu Y, Ai Z, Yang L, Gao Y, Du J et al. Vitamin $C$ enhances Nanog expression via activation of the JAK/STAT signaling pathway. Stem Cells 2014; 32 . 166-176.

13. Rhinn M, Dolle P. Retinoic acid signalling during development. Development 2012; 139 : 843-858.

14. Ribes V, Le Roux I, Rhinn M, Schuhbaur B, Dolle P. Early mouse caudal development relies on crosstalk between retinoic acid, Shh and Fgf signalling pathways. Development 2009; 136: $665-676$.

15. Kosik KS. The neuronal microRNA system. Nat Rev Neurosci 2006; 7: 911-920.

16. Kosik KS, Krichevsky AM. The elegance of the microRNAs: a neuronal perspective. Neuron 2005; 47: 779-782.

17. Cunningham TJ, Duester $\mathrm{G}$. Mechanisms of retinoic acid signalling and its roles in organ and limb development. Nat Rev Mol Cell Biol 2015; 16: 110-123.

18. Soprano DR, Teets BW, Soprano KJ. Role of retinoic acid in the differentiation of embryonal carcinoma and embryonic stem cells. Vitam Horm 2007; 75: 69-95.

19. Dhara SK, Stice SL. Neural differentiation of human embryonic stem cells. J Cell Biochem 2008; 105: 633-640.

20. Al Tanoury Z, Gaouar S, Piskunov A, Ye T, Urban S, Jost B et al. Phosphorylation of the retinoic acid receptor RARgamma2 is crucial for the neuronal differentiation of mouse embryonic stem cells. J Cell Sci 2014; 127(Pt 9): 2095-2105.

21. Ong SG, Lee WH, Kodo K, Wu JC. MicroRNA-mediated regulation of differentiation and trans-differentiation in stem cells. Adv Drug Deliv Rev 2015; 88: 3-15.

22. Schug TT, Berry DC, Shaw NS, Travis SN, Noy N. Opposing effects of retinoic acid on cell growth result from alternate activation of two different nuclear receptors. Cell 2007; 129: 723-733.

23. Tang XH, Retinoids Gudas LJ. Retinoic acid receptors, and cancer. Annu Rev Pathol 2011; 6 : 345-364.

24. Hu BY, Weick JP, Yu J, Ma LX, Zhang XQ, Thomson JA et al. Neural differentiation of human induced pluripotent stem cells follows developmental principles but with variable potency. Proc Natl Acad Sci USA 2010; 107: 4335-4340.

25. Maden M. Retinoic acid in the development, regeneration and maintenance of the nervous system. Nat Rev Neurosci 2007; 8: 755-765.

26. Shenoy A, Blelloch RH. Regulation of microRNA function in somatic stem cell proliferation and differentiation. Nat Rev Mol Cell Biol 2014; 15: 565-576.

27. Landgren $\mathrm{H}$, Carlsson P. FoxJ3, a novel mammalian forkhead gene expressed in neuroectoderm, neural crest, and myotome. Dev Dyn 2004; 231: 396-401.

28. Yamamoto H, Morino K, Nishio Y, Ugi S, Yoshizaki T, Kashiwagi A et al. MicroRNA-494 regulates mitochondrial biogenesis in skeletal muscle through mitochondrial transcription factor A and Forkhead box j3. Am J Physiol Endocrinol Metab 2012; 303 : E1419-E1427

29. Di Padova M, Caretti G, Zhao P, Hoffman EP, Sartorelli V. MyoD acetylation influences temporal patterns of skeletal muscle gene expression. J Biol Chem 2007; 282: 37650-37659

30. Verrier L, Escaffit F, Chailleux $C$, Trouche D, Vandromme M. A new isoform of the histone demethylase JMJD2A/KDM4A is required for skeletal muscle differentiation. PLoS Genet 2011; 7: e1001390.

31. Dugas JC, Cuellar TL, Scholze A, Ason B, Ibrahim A, Emery B et al. Dicer1 and miR-219 are required for normal oligodendrocyte differentiation and myelination. Neuron 2010; 65: $597-611$. 
32. Tay Y, Zhang J, Thomson AM, Lim B, Rigoutsos I. MicroRNAs to Nanog, Oct4 and Sox2 coding regions modulate embryonic stem cell differentiation. Nature 2008; 455: 1124-1128.

33. Gaughwin P, Ciesla M, Yang H, Lim B, Brundin P. Stage-specific modulation of cortical neuronal development by Mmu-miR-134. Cereb Cortex 2011; 21: 1857-1869.

34. Boyer LA, Lee TI, Cole MF, Johnstone SE, Levine SS, Zucker JP et al. Core transcriptional regulatory circuitry in human embryonic stem cells. Cell 2005; 122: 947-956.

35. Rohwedel J, Guan K, Wobus AM. Induction of cellular differentiation by retinoic acid in vitro. Cells Tissues Organs 1999; 165: 190-202.

36. Okada Y, Shimazaki T, Sobue G, Okano H. Retinoic-acid-concentration-dependent acquisition of neural cell identity during in vitro differentiation of mouse embryonic stem cells. Dev Biol 2004; 275: 124-142.

37. Ronn RE, Guibentif C, Moraghebi R, Chaves P, Saxena S, Garcia B et al. Retinoic acid regulates hematopoietic development from human pluripotent stem cells. Stem Cell Reports 2015; 4: 269-281.

38. Duester $\mathrm{G}$. Retinoic acid synthesis and signaling during early organogenesis. Cell 2008; 134 921-931.

39. Ohoka Y, Yokota A, Takeuchi H, Maeda N, Iwata M. Retinoic acid-induced CCR9 expression requires transient TCR stimulation and cooperativity between NFATC2 and the retinoic acid receptor/retinoid X receptor complex. J Immunol 2011; 186: 733-744.

40. Mark M, Ghyselinck NB, Chambon P. Function of retinoid nuclear receptors: lessons from genetic and pharmacological dissections of the retinoic acid signaling pathway during mouse embryogenesis. Annu Rev Pharmacol Toxicol 2006; 46: 451-480.

41. Janesick A, Wu SC, Blumberg B. Retinoic acid signaling and neuronal differentiation. Cell Mol Life Sci 2015; 72: 1559-1576.

42. Asano H, Aonuma M, Sanosaka T, Kohyama J, Namihira M, Nakashima K. Astrocyte differentiation of neural precursor cells is enhanced by retinoic acid through a change in epigenetic modification. Stem Cells 2009; 27: 2744-2752.

43. Kashyap V, Gudas LJ. Epigenetic regulatory mechanisms distinguish retinoic acid-mediated transcriptional responses in stem cells and fibroblasts. J Biol Chem 2010; 285 14534-14548.

44. Tang S, Huang G, Fan W, Chen Y, Ward JM, Xu X et al. SIRT1-mediated deacetylation of CRABPII regulates cellular retinoic acid signaling and modulates embryonic stem cell differentiation. Mol Cell 2014; 55: 843-855.

45. Kashyap V, Gudas LJ, Brenet F, Funk P, Viale A, Scandura JM. Epigenomic reorganization of the clustered Hox genes in embryonic stem cells induced by retinoic acid. J Biol Chem 2011; 286: 3250-3260.

46. Gudas LJ. Retinoids induce stem cell differentiation via epigenetic changes. Semin Cell Dev Biol 2013; 24: 701-705.

47. Kocerha J, Faghihi MA, Lopez-Toledano MA, Huang J, Ramsey AJ, Caron MG et al. MicroRNA-219 modulates NMDA receptor-mediated neurobehavioral dysfunction. Proc Nat Acad Sci USA 2009; 106: 3507-3512.
48. Santa-Maria I, Alaniz ME, Renwick N, Cela C, Fulga TA, Van Vactor D et al. Dysregulation of microRNA-219 promotes neurodegeneration through post-transcriptional regulation of tau. $J$ Clin Invest 2015; 125: 681-686.

49. Hudish LI, Blasky AJ, Appel B. miR-219 regulates neural precursor differentiation by direct inhibition of apical par polarity proteins. Dev Cell 2013; 27: 387-398.

50. Murai K, Sun G, Ye P, Tian E, Yang S, Cui $Q$ et al. The TLX-miR-219 cascade regulates neural stem cell proliferation in neurodevelopment and schizophrenia IPSC model. Nat Commun 2016; 7: 10965.

51. Wang Z, Yao H, Lin S, Zhu X, Shen Z, Lu G et al. Transcriptional and epigenetic regulation of human microRNAs. Cancer Lett 2013; 331: 1-10.

52. Wu H, Wang Y, Zhang Y, Yang M, Lv J, Liu J. TALE nickase-mediated SP110 knockin endows cattle with increased resistance to tuberculosis. Proc Natl Acad Sci USA 2015; 112: E1530-E1539.

53. Dupuy D, Bertin N, Hidalgo CA, Venkatesan K, Tu D, Lee D et al. Genome-scale analysis of in vivo spatiotemporal promoter activity in Caenorhabditis elegans. Nat Biotechnol 2007; 25: 663-668.

54. Prieto C, Risueno A, Fontanillo C, De las Rivas J. Human gene coexpression landscape: confident network derived from tissue transcriptomic profiles. PLOS ONE 2008; 3: e3911.

55. Barabasi AL, Oltvai ZN. Network biology: understanding the cell's functional organization. Nat Rev Genet 2004; 5: 101-113.

56. Ravasz E, Somera AL, Mongru DA, Oltvai ZN, Barabasi AL. Hierarchical organization of modularity in metabolic networks. Science 2002; 297: 1551-1555.

57. Carlson MR, Zhang B, Fang Z, Mischel PS, Horvath S, Nelson SF. Gene connectivity, function, and sequence conservation: predictions from modular yeast co-expression networks. BMC Genomics 2006; 7: 40 .

cc) (i) Cell Death and Disease is an open-access journal published by Nature Publishing Group. This work is licensed under a Creative Commons Attribution 4.0 International License. The images or other third party material in this article are included in the article's Creative Commons license, unless indicated otherwise in the credit line; if the material is not included under the Creative Commons license, users will need to obtain permission from the license holder to reproduce the material. To view a copy of this license, visit http://creativecommons.org/licenses/by/4.0/

(C) The Author(s) 2017

Supplementary Information accompanies this paper on Cell Death and Disease website (http://www.nature.com/cddis) 